\title{
Strange and charm particles at ZEUS
}

\author{
Volodymyr Aushev ${ }^{1}$ \\ (on behalf of the ZEUS Collaboration) \\ DESY/INR Notkestrasse 85, Hamburg 22607, Germany \\ E-mail: aushev@mail.desy.de
}

\begin{abstract}
The $\mathrm{K}$ s final state in ep collisions at HERA was studied with the ZEUS detector using an integrated luminosity of $0.5 \mathrm{fb}^{-1}$. Enhancements in the mass spectrum were observed and are attributed to the production of $\mathrm{f}_{2}(1270) / \mathrm{a}_{2}^{0}(1320), \mathrm{f}_{2}^{\prime}(1525)$ and $\mathrm{f}_{0}(1710)$. Masses and widths were obtained using a fit which takes into account theoretical predictions based on SU(3) symmetry arguments, and are consistent with the PDG values. The ${\underset{0}{0}}_{0}(1710)$ state, which has a mass consistent with a glueball candidate, was observed with a statistical significance of 5 standard deviations. The production of excited charm, $D_{1}(2420)^{0}$ and $D_{1}(2460)^{0}$, and charmstrange, $D_{s 1}(2536)$, mesons was measured with integrated luminosity of $126 \mathrm{pb}^{-1}$. Masses, widths and helicity parameters were determined. The measured yields were converted to the rates of $c$ quarks hadronising as a given excited charm meson and to the ratios of the dominant $D_{2}{ }^{*}(2460)$ and $D_{s 1}(2536)$ branching fractions. A search for the radially excited charm meson, $D^{* \prime}(2640)$, was also performed. The results are compared with those measured previously and with theoretical expectations.
\end{abstract}

European Physical Society Europhysics Conference on High Energy Physics EPS-HEP 2009, Krakow , Poland

July 16-22, 200

\footnotetext{
$1 \quad$ Speaker
} 


\section{Introduction}

Heavy-quark spectroscopy has recently undergone a renaissance with the discovery of several new states. The properties of these states challenge the theoretical description of heavyquark resonances. Therefore, further measurements of excited charm and charm-strange mesons are important.

Production of non-excited charm and charm-strange hadrons was extensively studied at HERA. The large charm production cross section at HERA also provides a means to study excited charm and charm-strange mesons produced in ep collisions. The results[3] of such study are reported in this report. It is restricted to decays, for which significant signals were identified: $\mathrm{D}_{1}(2420)^{0} \rightarrow \mathrm{D}^{*^{+}} \pi^{-}, \mathrm{D}_{2} *(2460)^{0} \rightarrow \mathrm{D}^{*^{+}} \pi^{-}, \mathrm{D}^{+} \pi^{-}, \mathrm{D}_{\mathrm{s} 1}(2536)^{+} \rightarrow \mathrm{D} *^{+} \mathrm{K}_{\mathrm{s}}^{0}, \mathrm{D} *^{0} \mathrm{~K}^{+}$

The corresponding antiparticle decays were also measured. Additionally a search for the radially excited charm meson, $\mathrm{D}^{*}(2640)^{+}$, in the $\mathrm{D}^{*+} \pi^{+} \pi^{-}$final state was also performed.

The Standart Model allows mesons with gluons, e.g. glueballs or hybrids. Lattice calculations predict that lightest glueball has $\mathrm{J}^{\mathrm{PC}}=0^{++}$and mass in range $1550-1750 \mathrm{MeV}$. $\mathrm{K}_{\mathrm{s}}{ }^{0} \mathrm{~K}_{\mathrm{s}}^{0}$ system couples to $\mathrm{J}^{\mathrm{PC}}=0^{++}, 2^{++}, 4^{++}$. I.e. $\mathrm{K}_{\mathrm{s}}{ }^{0} \mathrm{~K}_{\mathrm{s}}^{0}$ system is a good place for the lowest mass $0^{++}$glueball. Three enhancements corresponding to $f_{2}(1270) / a_{2}(1320), f_{2}{ }^{\prime}(1525)$ and $\mathrm{f}_{0}(1710)$ have been observed [3].

\section{Experimental set-up and event simulation.}

The analysis was performed using data taken with the ZEUS detector from 1995 to 2000. In this period, HERA collided electrons or positrons with energy $E_{\mathrm{e}}=27.5 \mathrm{GeV}$ and protons with energy $E_{p}=820 \mathrm{GeV}(1995-1997)$ or $E_{p}=920 \mathrm{GeV}(1998-2000)$. The data used in this analysis correspond to the full HERA luminosity of $0.5 \mathrm{fb}^{-1}\left(\mathrm{~K}_{\mathrm{s}}{ }^{0} \mathrm{~K}_{\mathrm{s}}^{0}\right.$ final states $)$ and integrated luminosity of $126.5 \mathrm{pb}^{-1}$ (the production of excited charm and charm-strange mesons ).

A detailed description of the ZEUS detector can be found elsewhere [1]. Charged particles were tracked in the central tracking detector (CTD), which operated in a magnetic field of $1.43 \mathrm{~T}$ provided by a thin superconducting solenoid. The CTD consisted of 72 cylindrical drift chamber layers, organized in nine superlayers covering the polar-angleз region $15^{\circ}<\theta<164{ }^{\circ}$. Before the HERAII running period the ZEUS tracking system was upgraded with a silicon Micro Vertex Detector (MVD).The high-resolution uranium-scintillator calorimeter (CAL) consisted of three parts: the forward (FCAL), the barrel (BCAL) and the rear (RCAL) calorimeters.

For study of the excited charm mesons Monte Carlo (MC) samples of charm and beauty events were produced with the Pythia 6.156 and Rapgap 2.0818 event generators. The Rapgap MC used Heracles 4.6.1 in order to incorporate first-order electroweak corrections. The generation included direct photon processes, in which the photon couples directly to a parton in the proton, and resolved photon processes, where the photon acts as a source of partons, one of which participates in the hard scattering process. The CTEQ5L and GRV LO parametrisations were used for the proton and photon structure functions, respectively. The charm and bottom quark masses were set to $1.5 \mathrm{GeV}$ and $4.75 \mathrm{GeV}$, respectively. The Lund string model as implemented in Jetset was used for hadronisation in Pythia and Rapgap. 


\section{K0sK0s resonance production}

The $\mathrm{K}_{\mathrm{s}}^{0}$ mesons were identified via their charged-decay mode, $\mathrm{K}_{\mathrm{s}}^{0} \rightarrow \pi^{+} \pi^{-}$. Both tracks

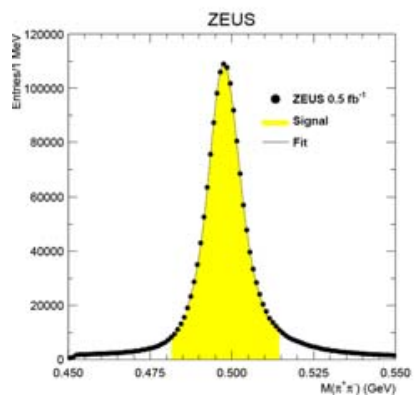
from the same secondary decay vertex were assigned the mass of the charged pion and the invariant mass of the charged of each pair was calculated. Events with at least two $\mathrm{K}_{\mathrm{s}}^{0}$ candidates were accepted for further analysis. In the mass range 481-515 $\mathrm{MeV}$ the number of candidates is about 1260000 . Figure 1 shows the invariant mass distribution of $\mathrm{K}_{\mathrm{s}}{ }^{0}$ candidates.

Fig.1 The measured invariant mass distribution for events with at least two candidates. The shaded area represents the signal window used for $\mathrm{K}_{\mathrm{s}}^{0} \mathrm{~K}_{\mathrm{s}}^{0}$ pair reconstruction.

Figure 2 shows the measured invariant-mass $\mathrm{K}_{\mathrm{s}}^{0} \mathrm{~K}_{\mathrm{s}}{ }^{0}$ spectrum. Three peaks are seen at

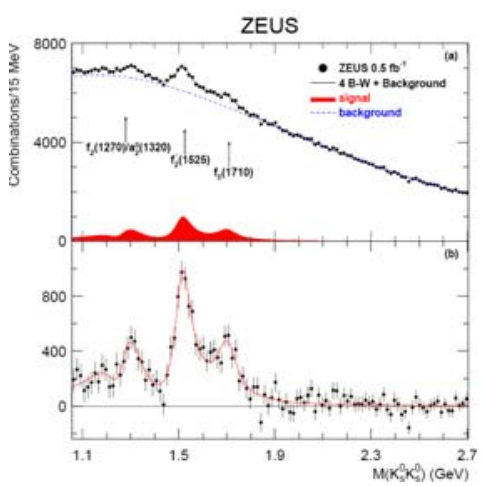
around 1300, 1500 and $1700 \mathrm{MeV}$. No state heavier than the $\mathrm{f}_{0}(1710)$ was observed. The invariant-mass distribution was fitted as a coherent sum of resonance production and the smoothly varying background.

Fig. 2. (a)The measured $\mathrm{K}_{\mathrm{s}}^{0} \mathrm{~K}_{\mathrm{s}}^{0}$ invariant-mass spectrum (dots). The solid line is the result of the coherent fit and dashed lines represents the background function.

(b)Background-subtracted invariant-mass spectrum; the result of the fit is shown as a solid line.

Two types of fit were used. The resonance masses and widths were allowed to vary in the fits. All results of the fits are shown in Table 1.

\begin{tabular}{|c|c|c|c|c|c|c|}
\hline Fit & \multirow{2}{*}{\multicolumn{2}{|c|}{$\begin{array}{c}\text { No interference } \\
96 / 95\end{array}$}} & \multirow{2}{*}{\multicolumn{2}{|c|}{$\begin{array}{c}\text { Interference } \\
86 / 97\end{array}$}} & \multirow{2}{*}{\multicolumn{2}{|c|}{ PDG 2007 Values }} \\
\hline$\chi^{2} / \mathrm{ndf}$ & & & & & & \\
\hline in $\mathrm{MeV}$ & ass & Width & Mass & Width & Mass & Width \\
\hline$f_{2}(1270)$ & \multirow{2}{*}{$1304 \pm 6$} & \multirow{2}{*}{$61 \pm 11$} & $1268 \pm 10$ & $176 \pm 17$ & $1275.4 \pm 1.1$ & $185.2_{-2.5}^{+3.1}$ \\
\hline$\overline{a_{2}^{0}(1320)}$ & & & $1257 \pm 9$ & $114 \pm 14$ & $1318.3 \pm 0.6$ & $107 \pm 5$ \\
\hline$f_{2}^{\prime}(1525)$ & $1523 \pm 3_{-8}^{+2}$ & $71 \pm 5_{-2}^{+17}$ & $1512 \pm 3_{-0.5}^{+1.4}$ & $83 \pm 9_{-4}^{+5}$ & $1525 \pm 5$ & $73_{-5}^{+6}$ \\
\hline$f_{0}(1710)$ & $1692 \pm 6_{-3}^{+9}$ & $125 \pm 12_{-32}^{+19}$ & $1701 \pm 5_{-2}^{+9}$ & $100 \pm 24_{-22}^{+7}$ & $1724 \pm 7$ & $137 \pm 8$ \\
\hline
\end{tabular}

Table 1. The measured masses and widths for $\mathrm{f}_{2}(1270), \mathrm{a}_{2}(1320), \mathrm{f}_{2}{ }^{\prime}(1525)$ and $\mathrm{f}_{0}(1710)$ states using decays as determined by one fit neglecting interference and another one with interference included. Both statistical and systematic uncertainties are quoted. Also quoted are the PDG values for comparison.

\section{Excited charm and charm strange production}

Events from both photoproduction and DIS were selected online with a three-level trigger. Events produced in the photoproduction regime with $\mathrm{Q}^{2}<1 \mathrm{GeV}^{2}$ contributed $70-80 \%$ of the selected $\mathrm{D}^{*^{+}}, \mathrm{D}^{+}$and $\mathrm{D}^{0}$ samples. The $\mathrm{D}^{*^{+}}$mesons were identified using the two decay channels $\mathrm{D}^{*^{+}} \rightarrow \mathrm{D}^{0} \pi_{\mathrm{s}}+\rightarrow\left(\mathrm{K}^{-} \pi^{+}\right) \pi_{\mathrm{s}}^{+}, \mathrm{D}^{*^{+}} \rightarrow \mathrm{D}^{0} \pi_{\mathrm{s}}+\rightarrow\left(\mathrm{K}^{-} \pi^{+} \pi^{+} \pi^{-}\right) \pi_{\mathrm{s}}{ }^{+}$. The pion from the $D^{*^{+}}$decays is referred to as the "soft" pion, $\pi_{\mathrm{s}}$, because it is constrained to have limited momentum by the 
small mass difference between the $\mathrm{D}^{*^{+}}$and $\mathrm{D}^{0}$. The mass difference for both channels was evaluated for all $\mathrm{D}^{*^{+}}$candidates (see Fig.3). Peaks at the nominal value of $M\left(\mathrm{D}^{*^{+}}\right)-M\left(\mathrm{D}^{0}\right)$ are evident.

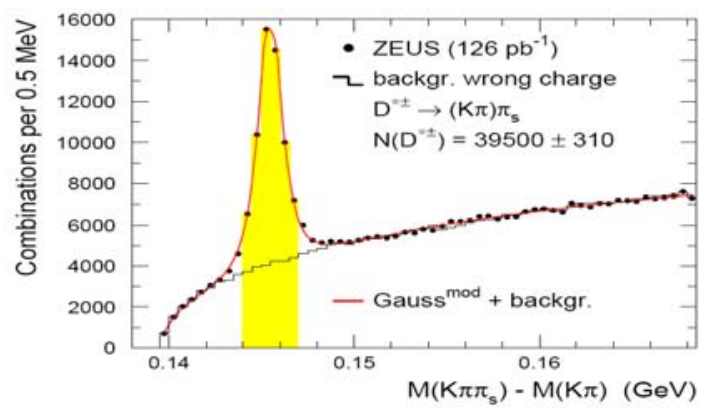

Fig. 3. The distributions of the mass differences (dots), $\Delta \mathrm{M}=\mathrm{M}(\mathrm{K} \pi \pi \mathrm{s})-\mathrm{M}(\mathrm{K} \pi)$ for $\mathrm{D}^{*} \rightarrow(\mathrm{K} \pi) \pi \mathrm{s}$ candidates and (similar for $\mathrm{D} * \pm$ $\rightarrow(\mathrm{K} \pi \pi \pi) \pi_{\mathrm{s}}$ candidates. The solid curves represent fits to the sum of a modified Gaussian function and a background function. The histograms show the $\Delta \mathrm{M}$ distributions for wrongcharge combinations. Only $\mathrm{D}^{*} \pm$ candidates from the shaded ranges were used for the analysis of excited states.

Figure 4 shows the $\mathrm{M}\left(\mathrm{D}^{*}{ }^{+} \pi_{\mathrm{a}}\right)$ distribution for $\mathrm{D} *^{+}$meson candidates reconstructed in both decay channels. A clear enhancement is seen in the range $2.4<\mathrm{M}\left(\mathrm{D}^{*} \pi_{\mathrm{a}}\right)<2.5$ $\mathrm{GeV}$, where contributions from $\mathrm{D}_{1}(2420)^{0}$ and $\mathrm{D}_{2} *(2460)^{0}$ mesons are expected. The wide $\mathrm{D}_{1}(2430)^{0}$ meson, which is also expected to contribute to the $\mathrm{M}\left(\mathrm{D}^{*}{ }^{+} \pi_{\mathrm{a}}\right)$ distribution, is not distinguishable from background due to its large width $(\sim 380 \mathrm{MeV})$.

No enhancement is seen in the $\mathrm{M}\left(\mathrm{D}^{*}{ }^{+} \pi_{\mathrm{a}}\right)$ distribution for wrong charge combinations

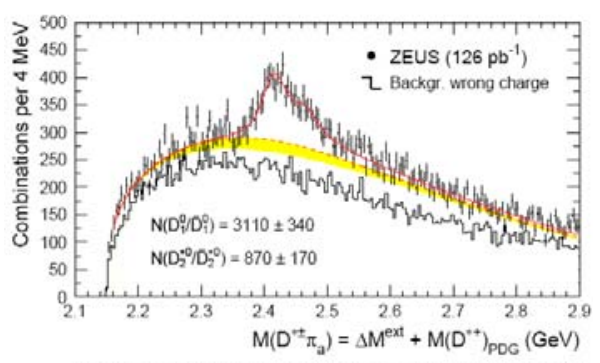
(histogram) formed by combining a $\mathrm{D}^{\text {, }}$ candidate and $\pi_{\mathrm{a}}$ with the same charges. To distinguish the $\mathrm{D}_{1}{ }^{0}$ and $\mathrm{D}_{2}{ }^{*}$ mesons from each other meson, the helicity angular distribution was used.

To reconstruct the decays $\mathrm{D}_{2} *^{0} \rightarrow \mathrm{D}^{+} \pi^{-}$an excited charm meson candidate was formed by combining each selected $\mathrm{D}^{+}$candidate with an additional track, assumed to be a pion $\left(\pi_{\mathrm{a}}\right)$, with a charge opposite to that of the $\mathrm{D}^{+}$.

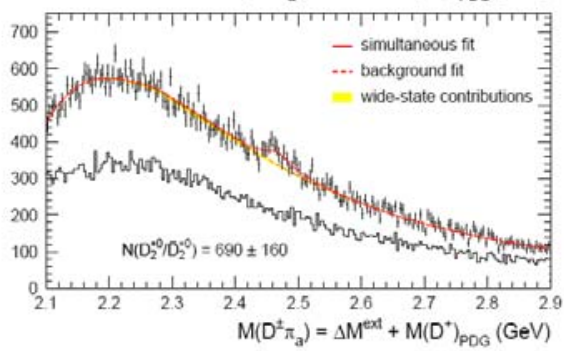

Fig.4. The distribution of (a) $\Delta \mathrm{Mext}+\mathrm{M}\left(\mathrm{D}^{*^{+}}\right) \mathrm{PDG}$, where $\Delta \mathrm{Mext}=\mathrm{M}\left(\mathrm{K} \pi \pi_{\mathrm{s}} \pi_{\mathrm{a}}\right)-\mathrm{M}\left(\mathrm{K} \pi \pi_{\mathrm{s}}\right)$ or $\Delta \mathrm{Mext}=\mathrm{M}\left(\mathrm{K} \pi \pi \pi \pi_{\mathrm{s}} \pi_{\mathrm{a}}\right)-$ $\mathrm{M}\left(\mathrm{K} \pi \pi \pi \pi_{\mathrm{s}}\right)$, for $\mathrm{D}_{1}{ }^{0}, \mathrm{D}_{2} *^{0} \rightarrow \mathrm{D}^{*+} \pi$ candidates and (b) $\mathrm{M}\left(\mathrm{D} \pm \pi_{\mathrm{a}}\right)=\Delta \mathrm{Mext}+\mathrm{M}\left(\mathrm{D}^{+}\right) \mathrm{PDG}$, where $\Delta \mathrm{Mext}=\mathrm{M}(\mathrm{K} \pi \pi \pi)-$ $\mathrm{M}(\mathrm{K} \pi \pi)$, for $\mathrm{D}_{2} *^{0} \rightarrow \mathrm{D}^{ \pm} \pi$ candidates (dots). The solid curves represent the result of the simultaneous fit with the background contribution.

The masses of the $\mathrm{D}_{1}{ }^{0}$ and $\mathrm{D}_{2} *^{0}$ were $2420.5 \pm 2.1$ (stat.) \pm 0.9 (syst.) $\mathrm{MeV}$ and 2469.1 \pm 3.7 (stat.) \pm 0.9 (syst.), respectively.

To reconstruct the $\mathrm{D}_{\mathrm{s} 1}{ }^{+} \rightarrow \mathrm{D}^{*} \mathrm{~K}_{\mathrm{S}}^{0}$ decays, a $\mathrm{D}_{\mathrm{s} 1}{ }^{+}$-meson candidate was formed by combining each selected $\mathrm{D}^{*^{+}}$candidate with the $\mathrm{K}_{\mathrm{S}}^{0}$ candidates reconstructed in the same event. Figure 5 shows the $\mathrm{M}\left(\mathrm{D} *+\mathrm{K}_{\mathrm{S}}^{0}\right)$ distribution for $\mathrm{D}^{*}{ }^{+}$meson candidates reconstructed in both decay channels. A clear signal is seen at the nominal value of $\mathrm{M}\left(\mathrm{D}_{\mathrm{s} 1}{ }^{+}\right)$. To reconstruct the $\mathrm{D}_{\mathrm{s} 1}{ }^{+}$ $\rightarrow \mathrm{D} *{ }^{0} \mathrm{~K}^{+}$decays, an excited charm-strange meson candidate was formed by combining each 
selected untagged $\mathrm{D}^{0}$ candidate with an additional track, assumed to be a kaon $\left(\mathrm{K}_{\mathrm{a}}\right)$, with a

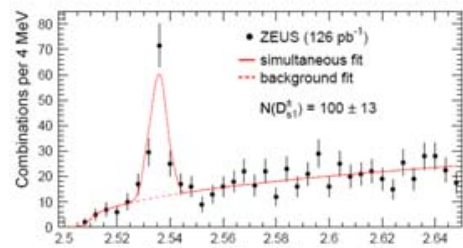

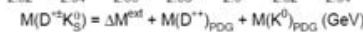

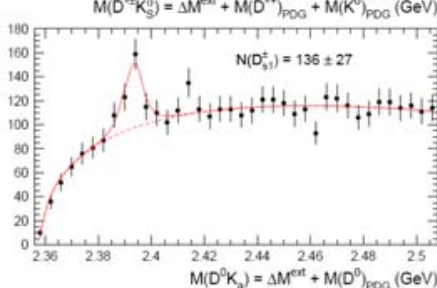
charge opposite to that of the particle taken as a kaon to form the $\mathrm{D}^{0}$ candidate. The mass was $\mathrm{M}\left(\mathrm{D}_{\mathrm{s} 1}{ }^{+}\right)=2535.57 \pm$ 0.44 (stat.) \pm 0.10 (syst.). The measured fragmentation fraction value is summarized in Table 2 and agrees with those obtained in $e+e-$ annihilations.

The ratios of the dominant $\mathrm{D}_{2}{ }^{* 0}$ and $\mathrm{D}_{\mathrm{s} 1}{ }^{+}$branching fractions are

$$
\begin{aligned}
& \left.\frac{\mathcal{B}_{D_{2}^{00} \rightarrow D^{+} \pi^{-}}}{\mathcal{B}_{D_{2}^{* 0} \rightarrow D^{*+} \pi^{-}}}=2.8 \pm 0.8 \text { (stat. }\right)_{-0.6}^{+0.5} \text { (syst.), } \\
& \frac{\mathcal{B}_{D_{s 1}^{+} \rightarrow D^{* 0} K^{+}}}{\mathcal{B}_{D_{s 1}^{+} \rightarrow D^{*+} K^{0}}}=2.3 \pm 0.6 \text { (stat.) } \pm 0.3 \text { (syst.) }
\end{aligned}
$$

Fig. 5. The distribution of the $\mathrm{M}\left(\mathrm{D}^{*+} \mathrm{K}_{\mathrm{S}}^{0}\right)$ and $\mathrm{M}\left(\mathrm{D}^{* 0} \mathrm{~K}^{+}\right)$mass.

To search for the $\mathrm{D}^{*}(2640)^{ \pm} \rightarrow \mathrm{D} *^{ \pm} \pi^{+} \pi^{-}$decays, a $\mathrm{D} *^{\prime}(2640)^{ \pm}$candidate was formed by combining each selected $\mathrm{D}^{*^{ \pm}}$candidate with two additional tracks with opposite charges.

\begin{tabular}{|c|c|c|c|}
\hline & $f\left(c \rightarrow D_{1}^{0}\right)[\%]$ & $f\left(c \rightarrow D_{2}^{* 0}\right)[\%]$ & $f\left(c \rightarrow D_{s 1}^{+}\right)[\%]$ \\
\hline \hline ZEUS & $3.5 \pm 0.4_{-0.6}^{+0.4}$ & $3.8 \pm 0.7_{-0.6}^{+0.5}$ & $1.11 \pm 0.16_{-0.10}^{+0.08}$ \\
\hline OPAL [38] & $2.1 \pm 0.7 \pm 0.3$ & $5.2 \pm 2.2 \pm 1.3$ & $1.6 \pm 0.4 \pm 0.3$ \\
\hline ALEPH [39] & & & $0.94 \pm 0.22 \pm 0.07$ \\
\hline Model [42] & 1.7 & 2.4 & 0.54 \\
\hline
\end{tabular}

Table 2. The fractions of c quarks harmonizing into the $\mathrm{D}_{1}{ }^{0}, \mathrm{D}_{2}{ }^{* 0}$ and Ds1+ mesons.

No radially excited $D *_{-}+$meson was observed. The upper limit $0.4 \%(95 \%$ C.L. $)$ is stronger than the $0.9 \%$ limit on the $\mathrm{D}^{*}(2640)^{ \pm}$production in charm fragmentation obtained by OPAL.

\section{Summary}

Observed three enhancements corresponding to $\mathrm{f}_{2}(1270) / \mathrm{a}_{2}(1320), \mathrm{f}_{2}{ }^{\prime}(1525)$ and $\mathrm{f}_{0}(1710)$. Masses and widths of $f_{2}^{\prime}(1525)$ and $f_{0}(1710)$ consistent with PDG; No state observed heavier than $\mathrm{f}_{0}(1710)$; Sizeable production of the excited charm and charm-strange mesons was observed. Measured masses of the $\mathrm{D}_{1}{ }^{0}, \mathrm{D}_{2} *^{0}$ and $\mathrm{D}_{\mathrm{s} 1}{ }^{+}$in reasonable agreement with the world average values. $\mathrm{D}_{1}{ }^{0}$ width $53.2 \mathrm{MeV}$ above PDG $20.4 \mathrm{MeV}$ and helicity parameter $\mathrm{h}=5.9$ consistent with prediction for pure D-wave. Measured $\mathrm{D}_{\mathrm{s} 1}{ }^{+}$helicity parameter $\mathrm{h}=-0.74$, inconsistent with prediction for a pure D- or S- waves. Suggests significant contributions of both waves. Ratios of dominant branching fractions are in agreement with the world average values. Fraction of c quarks into $\mathrm{D}_{10}$, are consistent with $\mathrm{D}_{2} *^{0}$ and $\mathrm{D}_{\mathrm{s} 1}{ }^{+}$obtained in e+ecollisions. No radially excited D*' $(2640)^{ \pm}$meson was observed.

\section{References}

[1] B. Baggins, ZEUS Coll., U. Holm (ed.), The ZEUS Detector. Status Report (unpublished), DESY (1993), available on http://www-zeus.desy.de/bluebook/bluebook.html.

[2] ZEUS collaboration, Chekanov et. al.,Phys. Rev. Lett. 101, 112003 (2008)

[3] ZEUS collaboration, Chekanov et. al.,Eur. Phys. J., C60, 25(2009) 